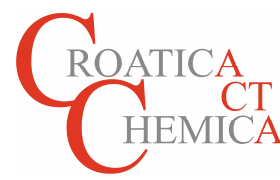

\title{
Surface Features of Nanocrystalline Alloys
}

\author{
Marcel Miglierini, ${ }^{1,2, *}$ Peter Matúš 3 \\ 1 Institute of Nuclear and Physical Engineering, Faculty of Electrical Engineering and Information Technology, Slovak University of Technology in Bratislava, \\ Ilkovičova 3, 81219 Bratislava, Slovakia \\ 2 Department of Nuclear Reactors, Faculty of Nuclear Science and Physical Engineering, Czech Technical University in Prague, V Holešovičkách 8, 180 00 Prague, \\ Czech Republic \\ 3 Institute of Laboratory Research on Geomaterials, Faculty of Natural Sciences, Comenius University in Bratislava, Mlynská dolina, Ilkovičova 6, 84215 \\ Bratislava, Slovakia \\ * Corresponding author's e-mail address: marcel.miglierini@stuba.sk
}

RECEIVED: October 6, 2015 * REVISED: January 30, 2016 * ACCEPTED: February 1, 2016

THIS PAPER IS DEDICATED TO DR. SVETOZAR MUSIĆ ON THE OCCASION OF HIS 70

\begin{abstract}
Nanocrystalline alloys are prepared by controlled annealing of metallic glass precursors. The latter are obtained by rapid quenching of a melt on a rotating wheel. This process leads to structural deviation of the produced ribbons' surfaces. Structural features of as-quenched and thermally annealed ${ }^{57} \mathrm{Fe}_{81} \mathrm{Mo}_{8} \mathrm{Cu}_{1} \mathrm{~B}_{10}$ ribbons were studied employing Conversion Electron Mössbauer Spectrometry (CEMS) and Conversion X-ray Mössbauer Spectrometry (CXMS). Enrichment of the alloy's composition in ${ }^{57} \mathrm{Fe} \mathrm{helped} \mathrm{in} \mathrm{identification} \mathrm{of} \mathrm{surface} \mathrm{crystallites} \mathrm{that}$ were formed even during the production process. Magnetite and bcc-Fe were found at the wheel side of the as-quenched ribbons whereas only bcc-Fe nanocrystals were uncovered at the opposite air side. Accelerated formation of bcc-Fe was observed in this side of the ribbons after annealing. The relative content of magnetite at the wheel side was almost stable in near surface areas (CEMS) and in more deep subsurface regions (CXMS). It vanished completely after annealing at $550^{\circ} \mathrm{C}$. No magnetite was observed at the air side of the ribbons regardless the annealing temperature and/or depth of the scanned regions.
\end{abstract}

Keywords: nanocrystalline alloys, CEMS, CXMS, surface crystallization.

\section{INTRODUCTION}

$\mathbf{M}$ etallic glasses (MGs) belong to a class of amorphous materials that, though introduced already couple of decades ago, are still attractive because of their unique magnetic ${ }^{[1]}$ as well as mechanical[ ${ }^{[2,3]}$ properties. Some MGs are used as precursors for preparation of nanocrystalline alloys (NCAs) that exhibit magnetic parameters superior to those of the original MGs. ${ }^{[4,5]}$ NCAs represent new class of nanocomposite materials the physical properties of which can be tailored not only by controlling their chemical composition but also by varying the size of crystalline grains, their morphology and structural arrangement. At present, three principal groups of NCAs are distinguish, namely FINEMET, ${ }^{[6]}$ NANOPERM, ${ }^{[7]}$ and HITPERM. ${ }^{[8]}$ Their chemical composition can be characterized as Fe-Si-Nb-Cu-B, Fe-M-(Cu)-B, and Fe,Co-M-(Cu)-B ( $\mathrm{M}=$ $\mathrm{Zr}, \mathrm{Mo}, \mathrm{Hf}, \mathrm{Ti}, \mathrm{Nb}, \ldots)$, correspondingly. Notably because of the presence of $\mathrm{Fe}$, they are routinely investigated by ${ }^{57} \mathrm{Fe}$ Mössbauer spectrometry which provides direct identification of all structurally different regions of the resonant nuclei that are located in nanocrystalline grains, residual amorphous matrix and between these two phases.

Fe-based NCAs attract a lot of scientific interest because, contrary to their amorphous counterparts, their magnetic parameters do not substantially deteriorate at elevated temperature during their practical exploitation. They can be easily prepared in a form of thin $(\sim 20 \mu \mathrm{m})$ ribbons by a method of planar flow casting of melt upon rotating quenching wheel and subsequent heat treatment. Due to the preparation procedure, both sides of the produced ribbons are fairly different from the structural point of view. They can consequently affect also formation of nanocrystalline grains when the as-quenched alloys are exposed to heat treatment. The ribbon's surfaces are henceforth denoted as the wheel and the air side for those 
parts of the ribbons which were in contact with the quenching wheel and the surrounding atmosphere, respectively.

In order to characterize the above mentioned distinctions, in this presentation we report on the use of surface sensitive Mössbauer effect techniques comprising Conversion Electron Mössbauer Spectrometry (CEMS) and Conversion X-Ray Mössbauer Spectrometry (CXMS) to follow structural variations that are observed at both sides of the ribbons. CEMS and CXMS provide information from subsurface regions that extend to the depth of $\sim 200 \mathrm{~nm}$ and $\sim 5 \mu \mathrm{m}$, respectively.

\section{EXPERIMENTAL DETAILS}

Ribbons of ${ }^{57} \mathrm{Fe}_{81} \mathrm{Mo}_{8} \mathrm{Cu}_{1} \mathrm{~B}_{10}$ MGs were prepared by the method of planar flow quenching of a melt upon rotating wheel. The casting was done in the air, that is, no protective atmosphere was used. The width and the thickness of the resulting ribbons were $1-2 \mathrm{~mm}$ and $\sim 20 \mu \mathrm{m}$, respectively. In order to facilitate the use of the CEMS and CXMS techniques, iron enriched to about $50 \%$ in the ${ }^{57} \mathrm{Fe}$ isotope was used for the production of this MG alloy. Annealing of the obtained as-quenched ribbons was performed for 30 min in a vacuum $\left(p \sim 10^{-3} \mathrm{~Pa}\right)$ at $370,410,450,510$, and $550^{\circ} \mathrm{C}$.

Due to the use of ${ }^{57} \mathrm{Fe}$, the total weight of the primary melt was limited to $\sim 1 \mathrm{~g}$. This turned out to be rather demanding as far as the absolute weights of the compositional elements were concerned. Thus, the resulting final chemical composition of the prepared asquenched ribbons was carefully checked. The content of Mo and B was determined by optical emission spectrometry with inductively coupled plasma (Jobin Yvon 70 Plus ICP spectrometer). Fe and Cu were established by flame atomic absorption spectrometry (Perkin Elmer 1100 spectrometer).

Mössbauer effect experiments were performed with conventional constant acceleration spectrometer using a ${ }^{57} \mathrm{Co} / \mathrm{Rh}$ source. Detection of conversion electrons and conversion $\mathrm{X}$-rays was ensured by gas counter using $\mathrm{He}+10 \% \mathrm{CH}_{4}$ and $\mathrm{Ar}+10 \% \mathrm{CH}_{4}$ gas mixtures, respectively, at room temperature. Velocity calibration was done using a thin $\alpha$-Fe foil (Goodfellow, thickness $12.5 \mu \mathrm{m}$, purity $99.85 \%$ ). Evaluation of the spectra was accomplished by the Confit software. ${ }^{[9]}$

Spectral parameters were refined using a fitting model that assumed presence of nanocrystalline grains embedded in a residual amorphous matrix. Details on the concept of this model can be found elsewhere. ${ }^{[10]}$ Here we describe only the assignment of the individual fitting components. Because of the alloy's composition, the amorphous matrix is paramagnetic in the as-quenched state (at room temperature) but exhibits also some magnetic interactions after annealing at higher temperatures when the alloy contains sufficiently high amount of ferromagnetic nanograins which polarize the originally paramagnetic amorphous matrix via ferromagnetic exchange interactions among them. Consequently, the amorphous parts of the Mössbauer spectra were fitted with distributions of quadrupole splitting and hyperfine magnetic fields, correspondingly. Intensities of the lines that correspond to the latter were free during the fit. The emerging bcc-Fe phase was represented by a Lorentzian-line sextet that was ascribed to the inner bulk of the nanograins. All its parameters including line intensities were free during the fitting procedure. According to the chosen fitting model[10] surfaces of bcc-Fe nanograins were characterised by distributions of hyperfine magnetic fields. Line intesities of the particular sextets were fixed to represent random orientation of magnetic moments due to broken symmetry of Fe atoms situated at the surfaces of the nanograins. Additional crystalline components, where applicable, were refined by sextets of Lorentizian lines without any restrictions.

\section{RESULTS AND DISCUSSION}

Mössbauer spectra of the as-quenched alloy show pronounced broad central signals. They are clearly seen in the insets of Figure 1 and Figure 2 where CEMS and CXMS spectra, respectively, are displayed in full scale. These

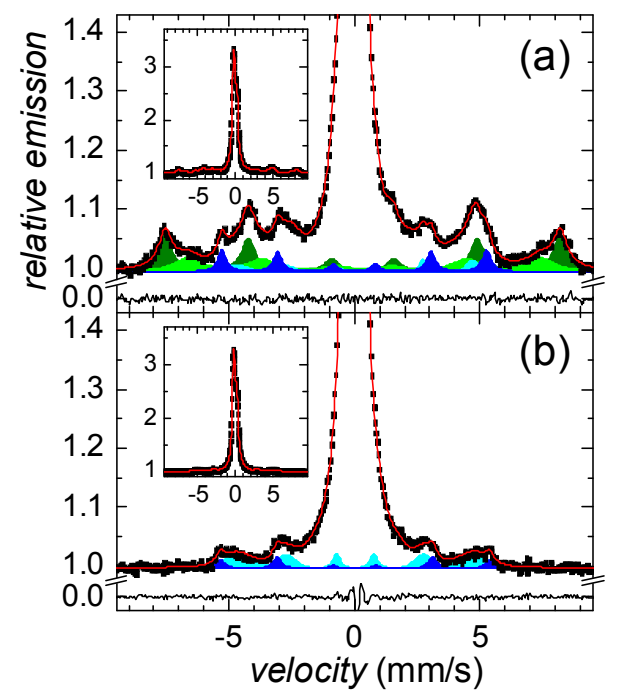

Figure 1. CEMS spectra of the as-quenched ${ }^{57} \mathrm{Fe}_{81} \mathrm{Mo}_{8} \mathrm{Cu}_{1} \mathrm{~B}_{10}$ ribbon taken from the wheel (a) and air side (b). Partial spectral components which belong to bccFe nanocrystals (blue), their surfaces (cyan), and magnetite (A-site dark green, B-site light green) are also plotted. The insets show CEMS spectra in full scale. 
parts of the spectra were refined with distributions of quadrupole splitting. The latter indicate that the investigated MGs are amorphous and paramagnetic at room temperature. Nevertheless, zoomed-out $y$-scales have unveiled presence of additional spectral lines that are seen outside the central broad peak. They were identified by the help of spectral parameters including hyperfine magnetic field and isomer shift and belong to quenched-in crystalline phases that were formed during the production process. One of the sextets belongs to bcc-Fe, plotted in blue colour, two sextets are assigned to $\mathrm{Fe}_{3} \mathrm{O}_{4}$ (A-site dark green, B-site light green), and the forth cyan component represents surface atoms of bcc-Fe nanocrystals via distributions of hyperfine magnetic fields. ${ }^{[10]}$

However, all the crystalline phases mentioned above are found only in the CEMS spectrum that was taken from the wheel side of the ${ }^{57} \mathrm{Fe}_{81} \mathrm{Mo}_{8} \mathrm{Cu}_{1} \mathrm{~B}_{10}$ asquenched ribbon shown in Figure 1a. The corresponding air side is magnetite-free and only small content of bcc-Fe nanocrystals is found here as demonstrated in Figure $1 \mathrm{~b}$. In deeper regions that are scanned by CXMS in Figure 2, negligible traces of spectral lines belonging to bcc-Fe and magnetite are visible only at the wheel side (Figure 2a) while the air side is fully amorphous and exhibits just a single broad central doublet in Figure $2 b$.

After annealing at rather moderate temperature of $370{ }^{\circ} \mathrm{C}$, the surface crystallization at the wheel side is almost the same within the CEMS detection region $(\sim 200 \mathrm{~nm})$ as in the as-quenched alloy. This is demon-

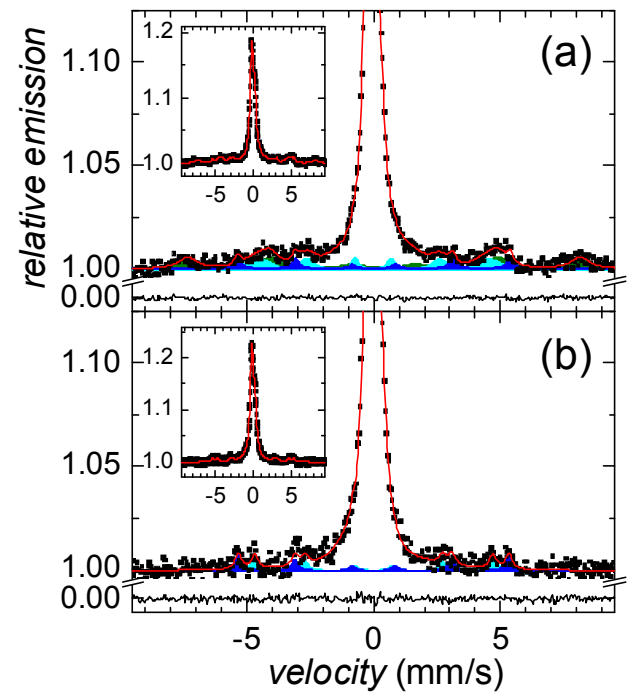

Figure 2. CXMS spectra of the as-quenched ${ }^{57} \mathrm{Fe}_{81} \mathrm{Mo}_{8} \mathrm{Cu}_{1} \mathrm{~B}_{10}$ ribbon taken from the wheel (a) and air side (b). Partial spectral components which belong to bcc-Fe nanocrystals (blue), their surfaces (cyan), and magnetite (dark green) are also plotted. The insets show CXMS spectra in full scale. strated in Figure 3a where, however a different scale of the $y$-axis is used as that in Figure 1a. On the other hand, looking at the air side CEMS spectrum in Figure $3 \mathrm{~b}$ one can notice pronounced progress of crystallization which is documented by significantly higher contribution of a magnetic sextet that belongs to bcc-Fe nanograins. Quantification of the amounts of particular spectral components is given below. This observation suggests that the onset of temperature-induced surface crystallization starts earlier at the air side of the investigated MG alloy than that at the wheel side.

Even more striking differences between the wheel and air sides of the ${ }^{57} \mathrm{Fe}_{81} \mathrm{Mo}_{8} \mathrm{Cu}_{1} \mathrm{~B}_{10}$ alloy annealed at $370^{\circ} \mathrm{C}$ are obvious from Figure 4 where the corresponding CXMS spectra are displayed. They provide information from the depth of about $5 \mu \mathrm{m}$. While the wheel side is nearly fully amorphous and only tiny traces of bcc-Fe sextet are hardly seen in Figure 4a (compare with Figure 2a), clearly visible sextuplet lines are evidenced in Figure 4b. They confirm that at the air side the crystallization progresses also into deeper subsurface regions. The presence of Mössbauer lines that indicate well developed crystallization is nicely seen also in the full CXMS spectrum that is shown in the inset of Figure $4 \mathrm{~b}$. Comparing Figure 3 and Figure 4 it is possible to conclude that the onset of crystallization is more accelerated at the air side of the ribbon than at the opposite, i.e. wheel side. One can speculate that it is because of better stabilization of the wheel side where the dissipation of excess heat during the

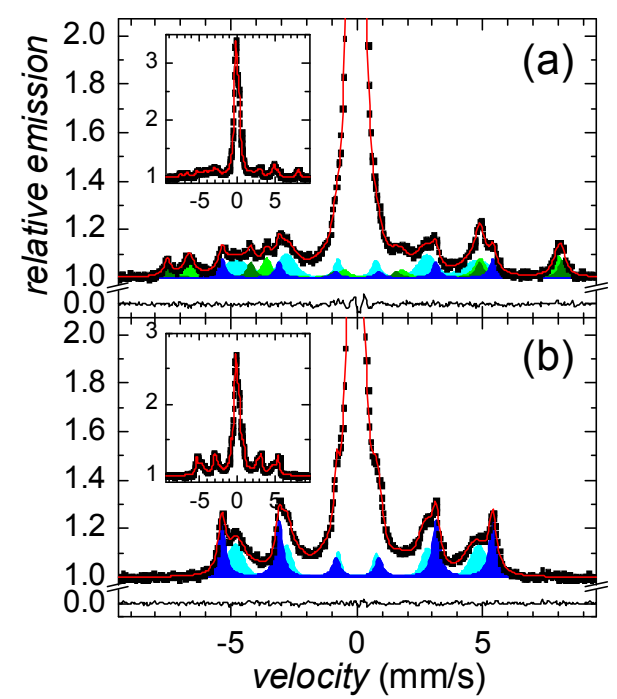

Figure 3. CEMS spectra of the ${ }^{57} \mathrm{Fe}_{81} \mathrm{Mo}_{8} \mathrm{Cu}_{1} \mathrm{~B}_{10}$ ribbon annealed at $370{ }^{\circ} \mathrm{C}$ taken from the wheel (a) and air side (b). Partial spectral components which belong to bcc-Fe nanocrystals (blue), their surfaces (cyan), and magnetite (A-site dark green, B-site light green) are also plotted. The insets show CEMS spectra in full scale. 
production process is more effective than at the air side. Consequently, even though the degree of quenched-in nucleation centres at the air side is effectively lower its smooth surface favours the progress of crystallization via Ostwald ripening. ${ }^{[11]}$

Finally, when the annealing temperature reaches $550{ }^{\circ} \mathrm{C}$, which is well beyond the onset of bulk crystalliza-

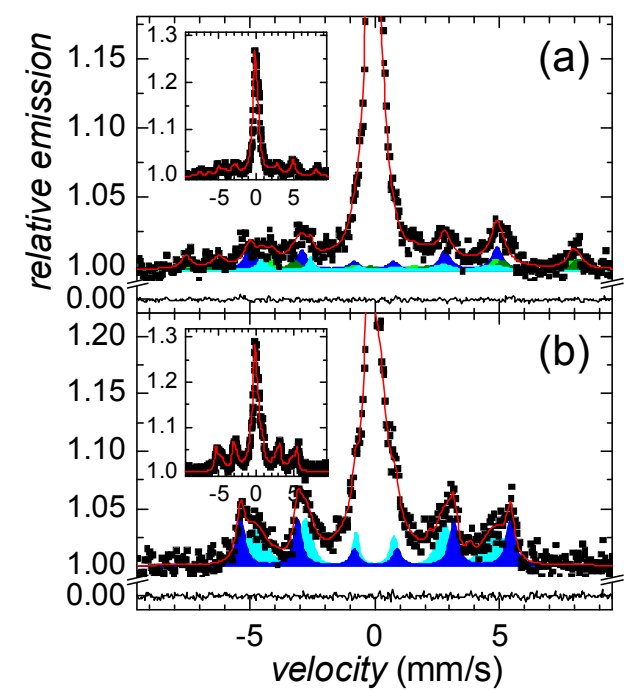

Figure 4. CXMS spectra of the ${ }^{57} \mathrm{Fe}_{81} \mathrm{Mo}_{8} \mathrm{Cu}_{1} \mathrm{~B}_{10}$ ribbon annealed at $370{ }^{\circ} \mathrm{C}$ taken from the wheel (a) and air side (b). Partial spectral components which belong to bcc-Fe nanocrystals (blue) and their surfaces (cyan) are also plotted. The insets show CXMS spectra in full scale.

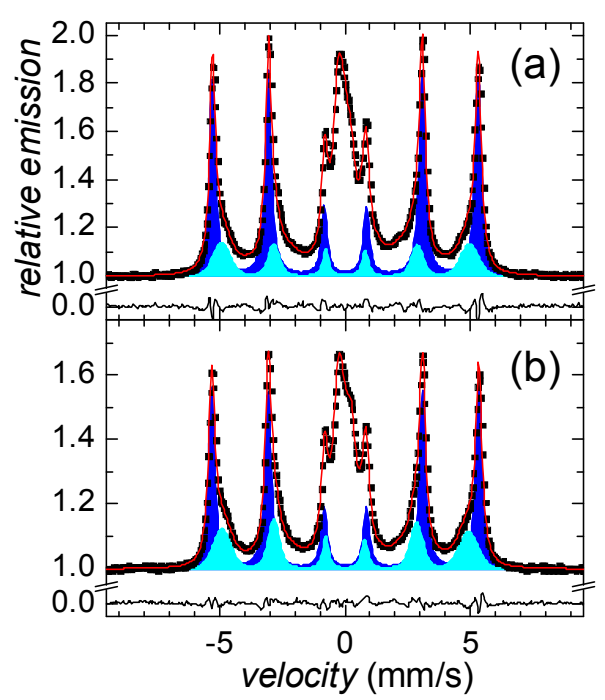

Figure 5. CEMS spectra of the ${ }^{57} \mathrm{Fe}_{81} \mathrm{Mo}_{8} \mathrm{Cu}_{1} \mathrm{~B}_{10}$ ribbon annealed at $550^{\circ} \mathrm{C}$ taken from the wheel (a) and air side (b). Partial spectral components which belong to bcc-Fe nanocrystals (blue) and their surfaces (cyan) are also plotted. tion $T_{x 1}\left(\sim 445^{\circ} \mathrm{C}\right),{ }^{[12]}$ all CEMS and CXMS spectra recorded from both sides of the ${ }^{57} \mathrm{Fe}_{81} \mathrm{Mo}_{8} \mathrm{Cu}_{1} \mathrm{~B}_{10} \mathrm{MG}$ ribbons look very alike in Figure 5 and Figure 6, respectively. The content of bcc-Fe nanograins is very similar and relatively high (> $60 \%$ ) though in the CEMS spectra (Figure 5b) their contribution is slightly higher at the air side than at the wheel side.

CXMS spectra in Figure 6 exhibit comparable behaviour. The amount of bcc-Fe is close to that in near subsurface regions scanned by CEMS. That is why we may conclude that at this annealing temperature the crystallization progresses from both sides of the ribbons into the bulk and differences in the Fe content become small. In addition, more abundant ferromagnetic Fe nanograins trigger formation of magnetic exchange interactions among them that consequently polarize the originally paramagnetic amorphous matrix. The latter starts to exhibit weak dipole magnetic hyperfine interactions. They are accounted for in the evaluation of the corresponding Mössbauer spectra by spectral components with distributed hyperfine magnetic fields. Their relative fraction gradually increases with rising temperature of annealing.

All Mössbauer spectra acquired by CEMS from both sides of the ${ }^{57} \mathrm{Fe}_{81} \mathrm{Mo}_{8} \mathrm{Cu}_{1} \mathrm{~B}_{10} \mathrm{MG}$ ribbons annealed the indicated temperatures including the as-quenched one are displayed in Figure 7. Those obtained by CXMS are depicted in Figure 8. The vertical dashed lines indicate positions of the spectral lines that belong to magnetite.

Practically no magnetite is present at the air side either in CEMS (Figure 7b) or in CXMS (Figure 8b) spectra. It

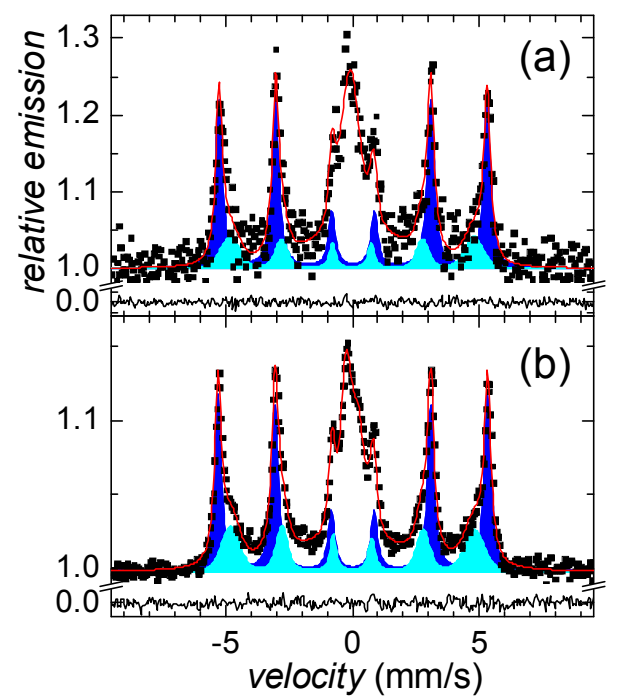

Figure 6. CXMS spectra of the ${ }^{57} \mathrm{Fe}_{81} \mathrm{Mo}_{8} \mathrm{Cu}_{1} \mathrm{~B}_{10}$ ribbon annealed at $550{ }^{\circ} \mathrm{C}$ taken from the wheel (a) and air side (b). Partial spectral components which belong to bcc-Fe nanocrystals (blue) and their surfaces (cyan) are also plotted. 
(a)

(b)
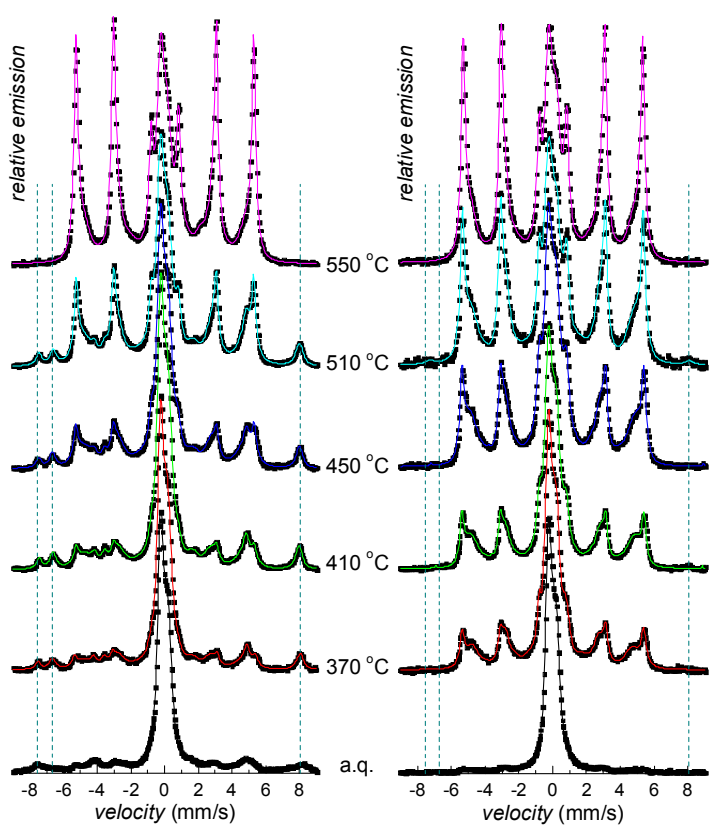

Figure 7. CEMS spectra of the ${ }^{57} \mathrm{Fe}_{81} \mathrm{Mo}_{8} \mathrm{Cu}_{1} \mathrm{~B}_{10}$ ribbons annealed at the indicated temperatures (a.q. = as-quenched) taken from the wheel (a) and air side (b). The vertical dashed lines show positions of Mössbauer lines of magnetite.

(a)

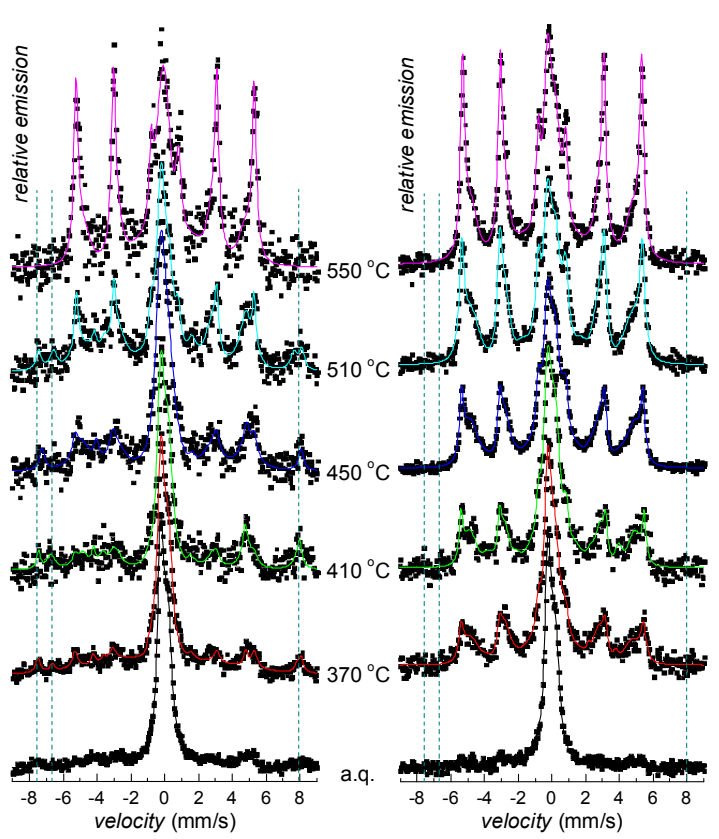

Figure 8. CXMS spectra of the ${ }^{57} \mathrm{Fe}_{81} \mathrm{Mo}_{8} \mathrm{Cu}_{1} \mathrm{~B}_{10}$ ribbons annealed at the indicated temperatures (a.q. = as-quenched) taken from the wheel (a) and air side (b). The vertical dashed lines show positions of Mössbauer lines of magnetite. was revealed only at the wheel side. As far as topography of this side is concerned, it contains many cavities. They were formed by air pockets which were squeezed between the wheel and the melt during the solidification process of the latter. We can speculate that magnetite, as a corrosion product, might be formed owing to residual air humidity that was trapped inside these cavities.

It is interesting to note, that although magnetite is found exclusively at the wheel side, it extends both into near surface areas (CEMS, Figure 7a) as well as into more deep subsurface regions (CXMS, Figure 8a) over the entire temperature range of heat treatment of the samples with the exception of annealing at $550{ }^{\circ} \mathrm{C}$ as discussed below. Relative fractions of the major structural components as derived from the CEMS and CXMS spectra of the annealed samples are depicted in Figure 9 and Figure 10, respectively.

In the parts (a) in Figures 9 and 10, the relative areas of magnetite (the total relative area of the spectral components related to the $A$ and $B$ sites) is plotted against temperature of annealing for both sides of the ribbons. Parts (b) in Figure 9 and Figure 10 exhibit evolution of the total crystalline phase (bcc-Fe + magnetite + bcc-Fe surfaces), which is plotted by squares, and the amorphous residual matrix (circles) for the wheel side (open symbols) and the air side (solid symbols). The corresponding error

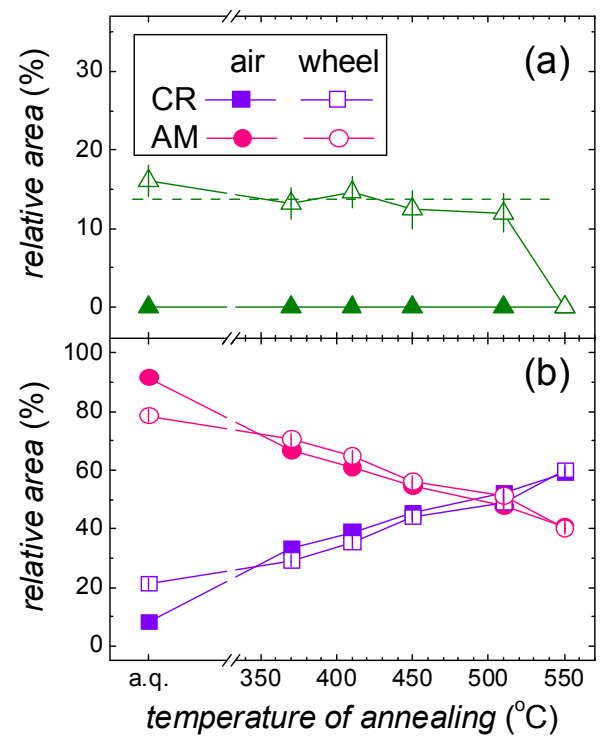

Figure 9. Relative area of spectral components derived from CEMS spectra of ${ }^{57} \mathrm{Fe}_{81} \mathrm{Mo}_{8} \mathrm{Cu}_{1} \mathrm{~B}_{10}$ ribbons plotted against temperature of annealing: part (a) - magnetite at the wheel side (green open triangles) and air side (green solid triangles), the dashed straight line represents the average value of magnetite component area; part (b) - total crystalline content (violet squares) and amorphous residual phase (pink circles) at the wheel side (open symbols) and air side (solid symbols). 


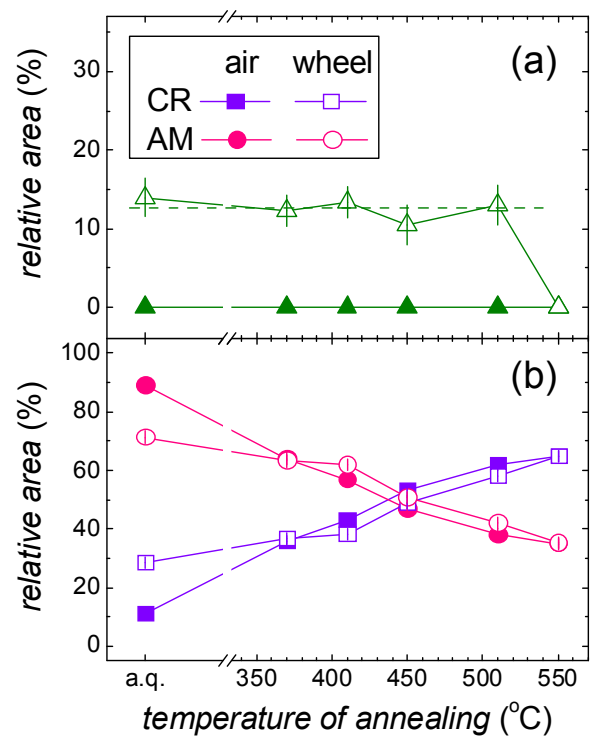

Figure 10. Relative area of spectral components derived from CXMS spectra of ${ }^{57} \mathrm{Fe}_{81} \mathrm{Mo}_{8} \mathrm{Cu}_{1} \mathrm{~B}_{10}$ ribbons plotted against temperature of annealing: part (a) - magnetite at the wheel side (green open triangles) and air side (green solid triangles), the dashed straight line represents the average value of magnetite component area; part (b) - total crystalline content (violet squares) and amorphous residual phase (pink circles) at the wheel side (open symbols) and air side (solid symbols).

bars in these parts of Figure 9 and Figure 10 are almost equal to the sizes of the symbols.

It is noteworthy that the presence of magnetite is unveiled only at the wheel side nearly for the whole range of annealing temperatures. The overall content of magnetite is slightly higher in near surface areas scanned by CEMS than in deeper regions (CXMS). Nevertheless, its content is almost stable. Average values of the corresponding spectral areas are plotted in Figure $9 a$ and Figure $10 \mathrm{a}$ by dashed straight lines. After heat treatment at $550{ }^{\circ} \mathrm{C}$, no traces of magnetite are revealed. Possible explanation of this observation is as follows. Magnetite, depending on its origin and the content of foreign ions, transforms to hematite in the temperature range $370-600{ }^{\circ} \mathrm{C} \cdot{ }^{[13]}$ As the transformation involves a change of structural rearrangement from ccp (cubic-close packing) to hcp (hexagonalclose packing), a comparatively high temperature is needed. At the same time, the size of the crystals should be taken into consideration. Because no signs of the Mössbauer sextet characteristic for hematite are found we assume that the newly formed crystals are very small and depict superparamagnetic behaviour. Consequently, the corresponding Mössbauer spectrum formed by a doublet would be hidden under the signal coming from the amorphous residual matrix.

\section{CONCLUSION}

Preparation of metallic glasses by rapid quenching on a rotating wheel is a common technique for production of amorphous ribbons. Nevertheless, it results qualitatively distinct surfaces on both ribbon's sides. Here, we have reported on surface behaviour of the ${ }^{57} \mathrm{Fe}_{81} \mathrm{Mo}_{8} \mathrm{Cu}_{1} \mathrm{~B}_{10}$ nanocrystalline alloy. In the as-quenched state, the original metallic glass has shown notable surface crystallization especially at the wheel side. The presence of magnetite and bcc-Fe was revealed. In the air side, only bcc-Fe nanocrystals were found. Occurrence of crystalline phases namely at the wheel side is unexpected because due to direct contact with the quenching wheel the dissipation of excess thermal energy is supposed to be better than at the air side of the produced ribbon.

After annealing, however, formation of bcc-Fe nanograins was accelerated quite rapidly at the air side while its progress was not so pronounced at the wheel side. Over extended range of annealing temperatures, only slightly higher amount of crystalline phases was found by CEMS in near surface areas located at the air side than at the opposite one. More deep subsurface regions scanned by CXMS show very alike crystalline contents at both sides of the ribbons. This can be interpreted in terms of evolution of the crystallization process in the bulk of the investigated nanocrystalline alloy.

Presence of magnetite, which was revealed owing to higher concentration of ${ }^{57} \mathrm{Fe}$ in the samples, is exclusively found only at the wheel side. Magnetite is presumably formed due to air humidity when during the production process air capsules are confined between the quenching wheel and the solidified melt. Subsequently, many cavities are formed in which corrosion of the metallic glass can start and as a result, magnetite occurs. During thermal treatment of the as-quenched alloy the relative contribution of magnetite is almost stable in both the near surface areas as evidenced by CEMS and in more deep subsurface regions (CXMS). Finally, at high enough annealing temperature of $550{ }^{\circ} \mathrm{C}$ magnetite fully transforms into hematite. Nevertheless, due to nanocrystalline nature and consequent superparamagnetic behaviour of the latter, its Mössbauer spectral lines are overlaid by those corresponding to the amorphous residual matrix.

Acknowledgment. The authors would like to thank D. Janičkovič for preparation of the samples. Financial support of the grant GACR 14-121449S is acknowledged.

\section{REFERENCES}

[1] G. Herzer, Acta Mater. 2013, 61, 718.

[2] A. Inoue, A. Takeuchi, Acta Mater. 2011, 59, 2243. 
[3] C. A. Schuh, T. C. Hufnagel, U. Ramamurty, Acta Mater. 2007, 55, 4067.

[4] K. Suzuki, A. Makino, A. Inoue, T. Masumoto, J. Appl. Phys. 1991, 70, 6232.

[5] G. Herzer, Phys. Scr. T 1993, 49A, 307.

[6] Y. Yoshizawa, A. Oguma, K. Yamauchi, J. Appl. Phys. 1988, 64, 6044.

[7] K. Suzuki, N. Kataoka, A. Inoue, A. Makino, T. Masumoto, Mater. Trans., JIM 1990, 31, 743.

[8] M. A. Willard, D. E. Laughlin, M. E. McHenry, D. Thoma, K. Sickafus, J. O. Cross, V. G. Harris, J. Appl. Phys. 1998, 84, 6773.
[9] T. Žák, Y. Jirásková, Surf. Interface Anal. 2006, 38, 710.

[10] M. Miglierini, J.-M. Grenèche, J. Phys.: Condens. Matter 1997, 9, 2303.

[11] M. Pavuk, M. Miglierini, M. Vujtek, M. Mashlan, R. Zboril, Y. Jiraskova, J. Phys.: Condens. Matter. 2007, 19, 216219.

[12] E. Illeková, D. Janičkovič, M Miglierini, I. Škorvánek, P. Švec, J. Magn. Magn. Mat. 2006, 304, e636.

[13] R. M. Cornell, U. Schwertmann, The Iron Oxides: Structure, Properties, Reactions, Occurrence and Uses, Wiley-Vch Verlag GmbH \& Co. KGaA, Weinheim, 2003, p. 346. 